\title{
Functions and mechanisms of intermittent negative pressure for osteogenesis in human bone marrow mesenchymal stem cells
}

\author{
ZHI YANG, JIAN-FENG YAO, PENG XU, JIAN-BING ZHANG, YU-MIN ZHANG, YANG-JUN ZHU, \\ SI-QING QIN, LIN LIU, HUI LIU, WEI-KUN HOU and KE XU \\ Department of Joint Surgery, Hong Hui Hospital, Xi'an Jiaotong University College of Medicine, \\ Xi'an, Shaanxi 710054, P.R. China
}

Received June 28, 2013; Accepted February 4, 2014

DOI: $10.3892 / \mathrm{mmr} .2014 .1968$

\begin{abstract}
The present study aimed to determine the mechanism by which low-intensity intermittent negative pressure affects the differentiation and proliferation of human mesenchymal stem cells (MSCs). Alkaline phosphatase (ALP) activity, type I collagen and vascular endothelial growth factor (VEGF) were detected to analyze differentiation. MTT and flow cytometry were employed to measure proliferation and apoptosis. Western blot analysis was used to examine endoplasmic reticulum (ER) stress-associated factors. This study was divided into two groups, including a normal group (without any treatment) and vacuum group (treated with a vacuum). There was a significant decrease in the proliferation of cells in the vacuum group. The number of cells in $\mathrm{S}$ phase was reduced significantly, while the rate of apoptosis and the activity of ALP were markedly increased under vacuum conditions. Expression of collagen type I and VEGF was significantly increased, and the ratio of osteoprotegrin to osteoprotegrin ligand was decreased significantly in the vacuum group. ER stress-associated proteins, p-PRKR-like ER kinase, inositol-requiring enzyme 1 and cleaved activating transcription factor 6 , as well as the downstream factors, were activated when treated with negative pressure. In conclusion, treatment with low-intensity and intermittent negative pressure may inhibit the proliferation of MSCs and trigger ER stress-associated cellular apoptosis, further enhancing osteogenesis activity and inducing differentiation to osteoblasts.
\end{abstract}

\section{Introduction}

Fractured bones require a long cycle of healing which results in patient suffering, prolonged hospitalization and increasing

Correspondence to: Professor Jian-Feng Yao, Department of Joint Surgery, Hong Hui Hospital, Xi'an Jiaotong University College of Medicine, Yan West Road 76, Xi'an, Shaanxi 710054, P.R. China E-mail: jianfengyao@yeah.net

Key words: negative pressure, marrow mesenchymal stem cells, proliferation, differentiation, endoplasmic reticulum stress economic costs for the individual and society. Bone healing may be delayed or obstructed by various numerous factors, including poor blood, loss of bone and soft tissue, metabolic disease, alcohol drinking, osteoporosis and high intraosseous pressure $(1,2)$. Therefore, in the clinic, the identification of methods to shorten the healing time and improve the healing rate of bone defects and osteonecrosis is important for bone healing.

Vacuum-associated closure (VAC) was first reported by Raffl (3) in 1952, when is was successfully applied to prevent infection and fluidity following flap transplantation. In the clinic, VAC has been used as an effective method to heal the soft tissue. However, its effects on bone tissue have never been investigated. Under specific circumstances, VAC may also promote rapid secondary wound healing by improving the blood supply (4). In soft tissue, VAC stretches cells, increases cell or tissue proliferation, promotes blood flow and reduces bacteria count, which helps to prevent cross infection, and the microenvironment of hypoxia and subacidity caused by VAC promotes angiogenesis and the influx of fibroblasts (5). The aforementioned backgrounds and function of VAC in bone tissue are investigated in the present study.

In a previous study, treatment of a compound fracture and the accompanying serious soft tissue injury by VAC was found to promote fracture union. However, the mechanism of VAC effects must be explored. In addition, the effect of negative pressure on bone healing must be investigated. Mesenchymal stem cells (MSCs) are multipotent stem cells that differentiate into a variety of cell types, including osteoblasts. The availability of MSCs is widespread and the cells are easy to isolate and culture. In modern bone repair clinics, MSCs have been employed as ideal seeding cells for substitution therapy.

The aim of the present study was to investigate the effects of negative pressure on the proliferation and differentiation of MSCs, and the specific mechanisms involved in these processes. Through this investigation, our understanding of the precise mechanisms of MSCs in bone regeneration therapy may be improved.

\section{Materials and methods}

MSC isolation and culture. Bone marrow tissue was collected from two patients diagnosed with hip osteoarthritis in the 
Department of Orthopaedics of The First Affiliated Hospital, Medical School of Xi'an Jiaotong University (Xi'an, China). A 5-ml tissue sample was harvested by aspiration from the femoral bone marrow cavity using heparin syringes, following osteotomy, during hip replacement surgery. The two patients had normal liver and kidney function and no metabolism osteodystrophy or infectious diseases. Consent was obtained from the patients prior to the sample selection in the study.

The collected bone marrow was put into centrifuge tubes, along with $2 \mathrm{ml} \mathrm{L-DMEM} \mathrm{medium} \mathrm{(Gibco-BRL,} \mathrm{Carlsbad,}$ CA, USA) and centrifuged at 1,200 x $\mathrm{g}$ for $5 \mathrm{~min}$ to remove the upper and medium fat droplets. The samples were then suspended in D-Hank's liquid, followed by separation on a Percoll gradient (Sigma, St. Louis, MO, USA) via centrifugation at 1,200 $\mathrm{x}$ g for $10 \mathrm{~min}$, then the middle layer of white film (mononuclear cells) was drawn and washed with D-Hank's liquid. The cells were then placed in L-DMEM medium in sterile culture bottles and cultured in an incubator at $37^{\circ} \mathrm{C}$ and $5 \%$ carbon dioxide. The third generation cells were digested with trypsin and $2 \times 10^{5}$ cells were incubated with antibodies against CD29, CD34, CD44, CD45 or HLA-DR, at room temperature for $30 \mathrm{~min}$ and washed twice with PBS. Next, cells were incubated with a secondary antibody labeled with FITC (Santa Cruz Biotechnology, Inc., Santa Cruz, CA, USA) for $15 \mathrm{~min}$ in the dark, followed by suspension in PBS following washing. Cells were examined via flow cytometry.

\section{2,3-bis-(2-methoxy-4-nitro-5-sulfophenyl)-2H-tetrazolium-} 5-carboxanilide (XTT) analysis. MSCs were digested and harvested using $0.25 \%$ trypsin (Wuhan Boshide Biotechnology, Wuhan, China) and 0.02\% EDTA and were plated in 96-well plates at a density of $1 \times 10^{4} / \mathrm{ml}$. At 1,3 and 5 days, $5 \mathrm{ml} \mathrm{XTT}$ (5 $\mathrm{mg} / \mathrm{ml}$ in PBS) was added to each well and the cells were incubated at $37^{\circ} \mathrm{C}$ in a humidified atmosphere containing $5 \%$ $\mathrm{CO}_{2}$. After 4-h incubation with XTT, the cells were lysed using a solution of $20 \%$ SDS and $50 \%$ DMSO at pH 4.7 and the absorbance was measured at $570 \mathrm{~nm}$ with an EL-311SX enzyme-linked immunosorbent assay reader (Bio-Tek Instruments, Winooski, VT, USA)

Detection of apoptosis. Cell apoptosis was detected by flow cytometry analysis that monitored annexin V-FITC binding and propidium iodide uptake simultaneously, according to the manufacturer's instructions (Sigma). The samples were analyzed by fluorescence on a FACSan flow cytometer (Beckman Coulter, Miami, FL, USA). The potential DNA fragmentation was examined by the TUNEL apoptosis detection kit (Chemicon, Temecula, CA, USA) following the manufacturer's instructions. Apoptotic bodies were stained by brilliant blue.

Quantitative PCR ( $q P C R)$. PCR was performed according to methods described previously (3) using the following primer sequences: Osteoprotegrin (OPG) forward, 5'-AATCAACTCAAAAATGTGGAATAGATGT-3' and reverse, 5'-GCGTAAACTTTGTAGGAACAGCAA-3'; Osteoprotegrin ligand (OPGL) forward, 5'-GCGTAAACT TTGTAGGAACAGCAA-3' and reverse, 5'-AACCAT GAGCCATCCACCAT-3'; osteocalcin forward, 5'-GGCAGTAAGGTGGTGAATAG-3' and reverse, 5'-TCCTGGAAGCCAATGTG-3'; and $\beta$-actin forward,
5'-AGGCACCAGGGCGTGAT-3' and reverse, 5'-TCGTCCCAGTTGGTGACGAT-3'.

Alkaline phosphatase (ALP) activity. Following plating at a density of $5 \times 10^{3}$ cells $/ \mathrm{cm}^{2}$ in 6 -well plates, cells were treated for 2 weeks, followed by solubilization of cellular proteins with $1 \%$ Triton-X in $0.9 \% \mathrm{NaCl}$ and centrifugation at $12,000 \mathrm{x} \mathrm{g}$ for $10 \mathrm{~min}$. The supernatants were assayed for ALP activity (Pointe Scientific, Detroit, MI, USA). One unit was defined as the activity that produces $1 \mathrm{nmol} p$-nitrophenol in $30 \mathrm{~min}$. The protein concentrations were determined with a Bio-Rad protein assay kit (Bio-Rad, Hercules, CA, USA) and ALP activity was normalized to the cellular protein contents.

Type I collagen and vascular endothelial growth factor (VEGF) detection. When the MSCs were treated under VAC conditions for 2 weeks, cells in slices were fixed for $20 \mathrm{~min}$ in $4 \%$ paraformaldehyde fluid. The primary antibodies were mouse anti-human type I collagen and VEGF monoclonal antibodies (Wuhan Bioshide Biotechnology). The cells were washed with PBS, blocked with goat serum and incubated with the primary antibody at a dilution of $1: 100$ at $4^{\circ} \mathrm{C}$ overnight. Following washing with PBS, the cells were incubated with the secondary antibody (Santa Cruz Biotechnology, Inc.) at $37^{\circ} \mathrm{C}$ for $30 \mathrm{~min}$. Next, the $A B C$ regents were incubated at room temperature for 20 min, washed with PBS and stained using DAB.

Western blot analysis of endoplasmic reticulum (ER) stress. In order to investigate the mechanism of MSC apoptosis, several ER stress-associated factors were detected using this assay, including p-PRKR-like ER kinase (PERK), inositol-requiring enzyme 1 (IRE-1) and activating transcription factor 6 (ATF6). These proteins are the main factors of the unfolded protein response (UPR) pathway. The downstream factors, which may trigger cell apoptosis, were also detected. A western blot analysis method was employed to analyze the ER stress factors.

The MSC lysates were separated by $15 \%$ SDS-PAGE and electrotransferred onto nitrocellulose membranes. Following blocking with $5 \%$ skimmed milk in phosphate buffered saline Tween-20 overnight at $4^{\circ} \mathrm{C}$, the membranes were incubated with goat pAb anti-human CHOP $(1: 2,000)$, mouse mAb anti-human p-Perk $(1: 3,000)$, mouse $\mathrm{mAb}$ anti-human IRE1 (1:2,000), mAb anti-human $\beta$-actin (1:600) (all Santa Cruz Biotechnology, Inc.), pAb anti-human caspase $3(1: 1,000)$ (Santa Cruz Biotechnology, Inc.), mAb anti-full length $(1: 1,000)$ and spliced XBP1 (Stressgen, Farmingdale, NY, USA), mAb anti-full length $(1: 2,000)$ and cleaved ATF6, anti-eIF2- $\alpha(1: 2,000)$ (both Santa Cruz Biotechnology, Inc.) for $2 \mathrm{~h}$ at room temperature. Next, membranes were incubated with horseradish peroxidase-conjugated anti-mouse (1:5,000), anti-rabbit or anti-goat IgG $(1: 2,000)$ (all Santa Cruz Biotechnology, Inc.). The reactive signals were visualized by an ECL kit (PE Applied Biosystems, Foster City, CA, USA).

Statistical analysis. Quantitative analysis of immunoblot images was performed using computer-assisted software Image Total Tech (Pharmacia, New York, NY, USA). Briefly, the image of the immunoblot was scanned with Typhoon (Pharmacia), digitalized and saved as TIF format. The values of each target blot were evaluated. Data are presented as 
A

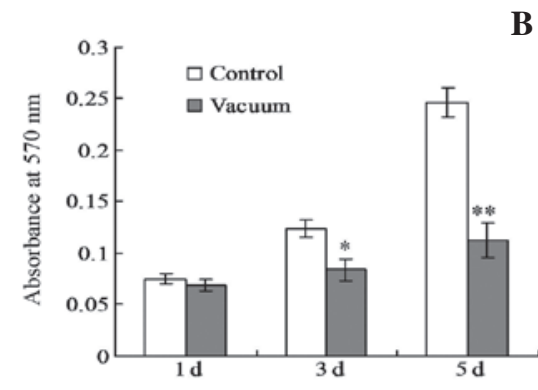

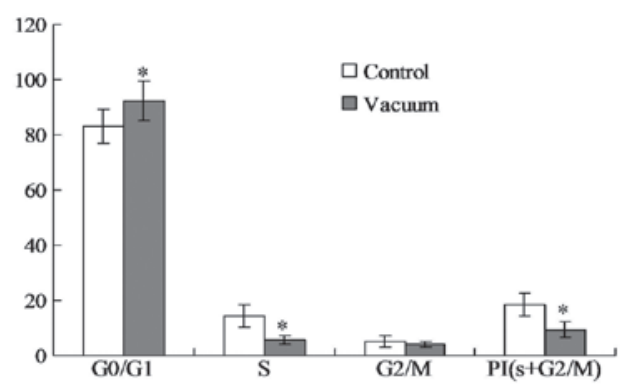

C
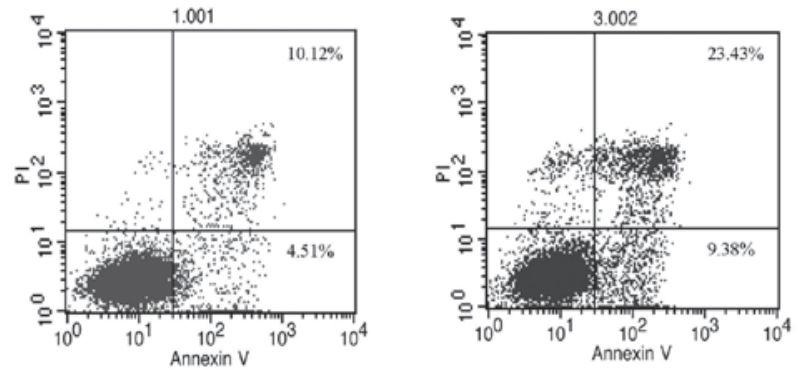

Figure 1.Cellular proliferation, cell cycle state and apoptosis analysis. (A) Cellular proliferation measured by the XTT assay. (B) Cell cycle state determined using flow cytometry. (C) Detection of apoptotic cells by flow cytometry. MSCs were treated for 2 weeks and double stained with annexin V and proidium iodide to detect early and late apoptotic changes in the cell membranes. ${ }^{*} \mathrm{P}<0.05$ and ${ }^{* * *} \mathrm{P}<0.01$, vs. control group. XTT, (2,3-bis-(2-methoxy-4-nitro-5-sulfophenyl)-2H-tetrazolium-5-carboxanilide); MSC, mesenchymal stem cells.

A

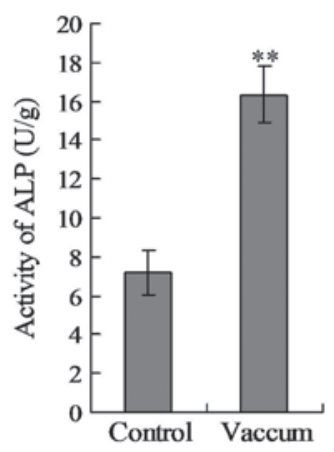

B

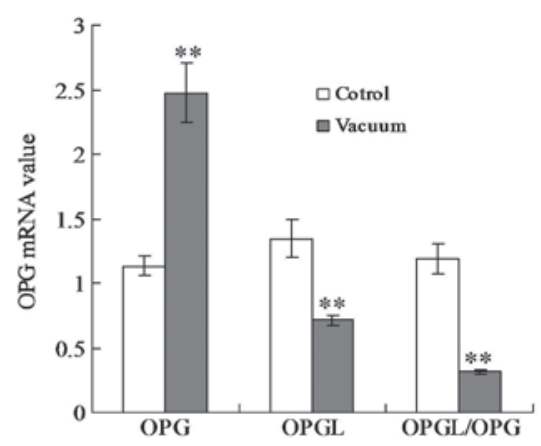

Figure 2. Detection of MSC differentiation. (A) Activity of ALP in the treated MSCs. (B) OPG and OPGL mRNA expression. ${ }^{*} \mathrm{P}<0.05$ and ${ }^{* * *} \mathrm{P}<0.01$, vs. control group. ALP, alkaline phosphatase; MSC, mesenchymal stem cells; OPG, osteoprotegerin; OPGL, OPG ligand.

the mean \pm standard deviation. A statistical analysis was performed using a t-test. $\mathrm{P}<0.05$ was used to indicate a statistically significant difference.

\section{Results}

Proliferation and apoptosis of negative pressure-treated $M S C s$. Using the flow cytometry analysis, cells were observed to be homogenous and, as is appropriate for MSCs, expressed CD29, CD34, CD44, CD45 and HLA-DR (data not shown). The XTT analysis results indicated that, in the experimental group, the proliferation of MSCs was reduced significantly compared with the control group (Fig. 1A; $\mathrm{P}<0.05$ ). Flow cytometry detection revealed that the apoptosis rate of the negative pressure-treated group (vacuum) was significantly higher compared with that of the control group (Fig. 1B). Furthermore, the percentage of cells in the $\mathrm{S}$ phase of the cellular cycle in the negative pressure-treated group was decreased significantly compared with the control group (Fig. 1C).

MSCs differentiation. When the MSCs were treated with negative pressure for 2 weeks, the activity of ALP in the vacuum group $(16.34 \pm 1.47 \mathrm{U} / \mathrm{g})$ was significantly higher compared with that of the control group $(7.19 \pm 1.18 \mathrm{U} / \mathrm{g})$ (Fig. 2A; $\mathrm{P}<0.01)$.

The qPCR results illustrated that the OPG mRNA transcription of the control group was significantly increased compared with the vacuum group (Fig. $2 \mathrm{~B}$; $\mathrm{P}<0.01$ ). By contrast, OPGL mRNA transcription was significantly decreased $(\mathrm{P}<0.01)$. The ratio of OPGL to OPG mRNA transcription reduced significantly in the vacuum group (Fig. 2B; $\mathrm{P}<0.01)$.

Immunohistochemical staining of type I collagen and $V E G F$. The immunohistochemical staining results indicated that type I collagen was not expressed in the control group 
A

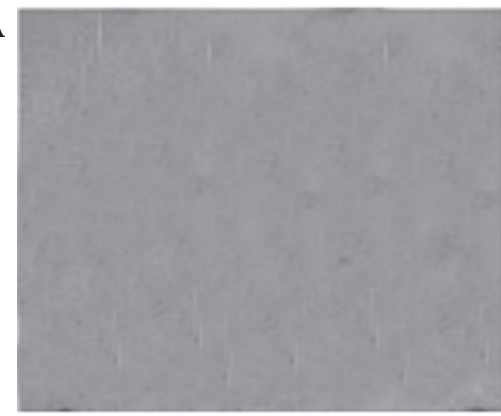

C

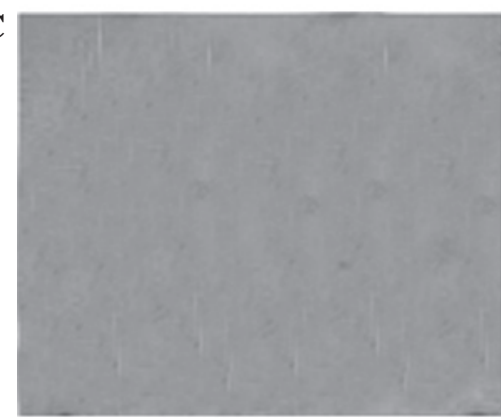

B

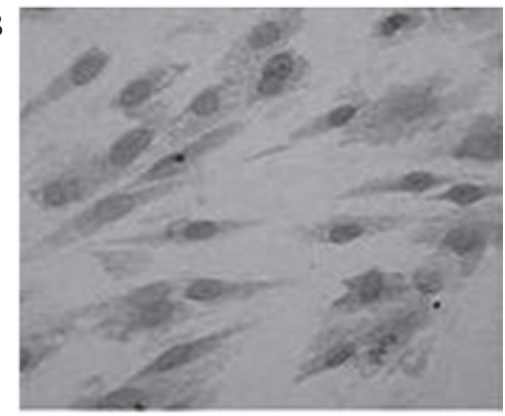

D

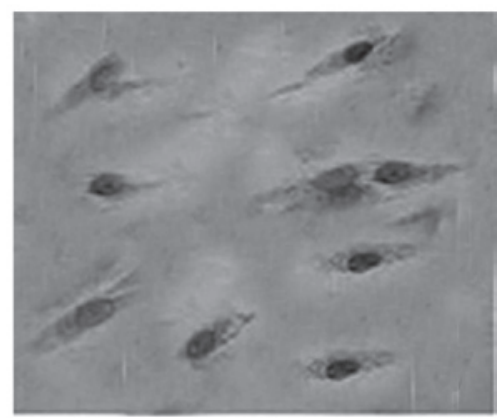

Figure 3. Immunohistochemical staining for collagen type I and VEGF of MSCs (SABC; magnification, x200). Immunohistochemical staining of MSCs revealed that expression of type I collagen was absent in (A) the control group but positive in (B) the negative pressure group, as a large number of yellow-brown particles appeared in cellular endochymema. In addition, the expression of VEGF in (C) the control group was weaker compared with that in (D) the negative pressure group. VEGF, vascular endothelial growth factor; MSC, mesenchymal stem cell; SABC, streptavidin-biotin complex.

A

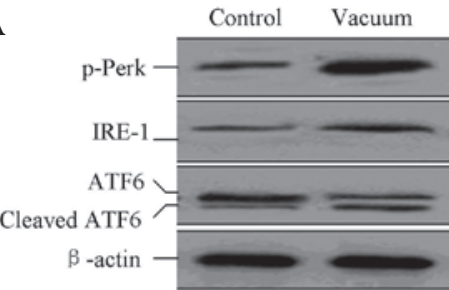

B

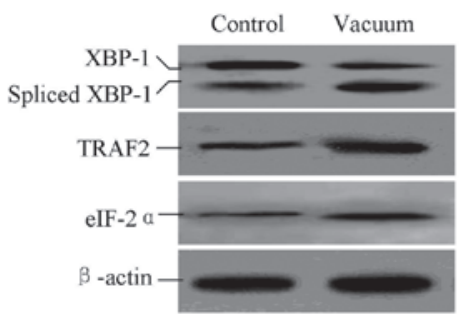

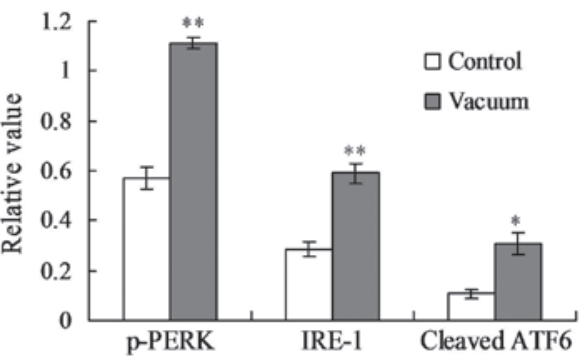

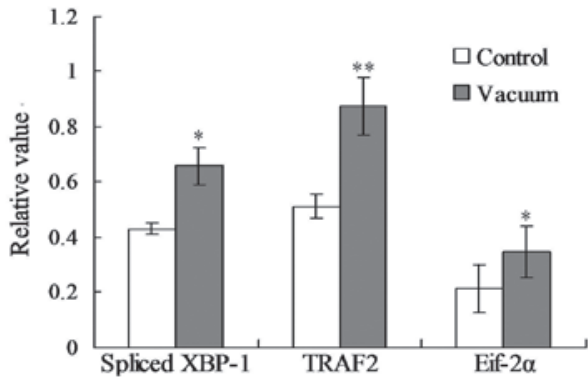

Figure 4. Detection of the UPR pathway proteins. (A) Detection of IRE1, p-Perk and cleaved ATF6. (B) Detection of the downstream TRAF2 protein, eIF-2 $\alpha$ protein and spliced XBP1 protein. Protein expression was calculated by the gray numerical value of each specific product versus that of $\beta$-actin. The average data of each preparation are evaluated based on three independent reactions and represented as the mean \pm standard deviation. ${ }^{*} \mathrm{P}<0.05$ and ${ }^{* *} \mathrm{P}<0.01$, vs. control group. UPR, unfolded protein response; ATF6, activating transcription factor 6; TRAF2, TNF receptor-associated factor 2; eIF-2 $\alpha$, eukaryotic translation initiation factor $2 \alpha$; XBP1, X-box binding protein 1; p-PERK, p-PRKR-like ER kinase; IRE-1, inositol-requiring enzyme 1.

(Fig. 3A), but was positively expressed in the vacuum group as a large number of yellow brown particles in the cellular hyaloplasm in those cells (Fig. 3B). In addition, the expression of VEGF in the vacuum group was stronger compared with the control group (Fig. 3A and B).

UPR pathway is involved in the inhibition of MSC proliferation. To explore the mechanisms of inhibition of proliferation in the negative pressure-treated MSCs, the main UPR factors, p-Perk, IRE-1 and ATF6, were detected by western blot analysis. As shown in Fig. 4A, the treatment of negative pressure triggered the phosphorylation of Perk (p-Perk), the cleavage of ATF6 protein and the activation of IRE-1. The protein expression of p-Perk, cleaved ATF6 and IRE-1 was significantly enhanced by the negative pressure treatment compared with the control group (Fig. 4A; $\mathrm{P}<0.05$ ). 
In addition, the downstream ER stress-associated proteins of the UPR pathway were detected, including the spliced X-box binding protein 1 (XBP1), TNF receptor-associated factor 2 (TRAF2) and eukaryotic initiation translation factor $2 \alpha(\mathrm{eIF}-2 \alpha)$ proteins. In the negative pressure treatment group, the levels of spliced XBP1, TRAF2 and eIF-2 $\alpha$ proteins were significantly increased compared with those of the control group (Fig. 4B; $\mathrm{P}<0.05$ ).

\section{Discussion}

In the clinic, the unique features of the negative pressure therapy method have been hypothesized to contribute to an optimized wound environment, including edema reduction, stimulation of angiogenesis and local blood flow, interstitial fluid flow and exudate management. The method also affects wound perfusion, growth factor, cytokine expression and cellular activity. These roles lead to enhanced granulation tissue formation and improved wound healing parameters (6-8). Therefore, the treatment should be focused on inducing osteogenesis through the effects of mechanical stimulation and growth factors in order to increase the early blood supply and restart bone healing (9). However, its effects on bone tissues and functions in bone repair are poorly understood. In the present study, the effects of negative pressure on osteogenesis in human MSCs and the specific mechanisms were investigated.

For our clinical practice, VAC cured soft tissue defects when applied at a pressure of $-50 \mathrm{kPa}$ for $30 \mathrm{~min}$ at a frequency of 2 times per day. In the compound injuries, bone healing was also improved when treated with negative pressure. We hypothesized that the negative pressure treatment may promote bone healing through mechanical stimulation, by increasing blood supply, recruitment of osteogenitor cells and inhibiting the proliferation of the osteogenitor cells (MSCs). MSCs are usually used as the seeding cells for gene and cell therapy of skeletal diseases, owing to their potential for differentiation into osteogenitor cells $(10,11)$. The oxygen concentration in vitro culture is $\sim 20 \%$, whereas in vivo oxygen concentration may decrease to only $0.4 \%-4.7 \%$ (12). Therefore, in the present study, the intermittent vacuum incubator (with $2 \%$ oxygen tension) was selected to incubate the MSCs with only a few cell cultures, including DMEM. Previous studies have indicated that the disorders correlated to bone repair often appear in older patients or those in which the ability of cells to proliferate has decreased $(13,14)$. Therefore, the target cells (MSCs) from the older patients were selected, and the effects of apoptosis on the proliferation of MSCs were investigated.

The present study also illustrated that the proliferation of MSCs was inhibited under the low-intensity and intermittent negative pressure. This inhibition may be attributed to the temporal hypoxia caused by negative pressure and the apoptosis caused by specific stress stimuli for the MSCs. A number of factors may affect the differentiation and proliferation of the MSCs, including mechanical stimulation and apoptotic responses. In the present study, the MSCs in the vacuum group exhibited significantly higher levels of apoptosis compared with the control group $(\mathrm{P}<0.05)$. The apoptosis inhibited the proliferation of the MSCs and triggered differentiation into osteoblasts in a subsequent step $(15,16)$. Our previous study also indicated that negative pressure may lead to upregulation of HIF-1, which contributes to the maintenance of oxygen homeostasis in physiological or pathological conditions. HIF-1 plays a significant role in the regulation of acute and intensive hypoxia $(17,18)$. Towler $(19)$ demonstrated that HIF-1 signaling in the development and differentiation of osteoblasts was central to the coupling of angiogenesis and long bone development in mice, and thus strategies promoting HIF-1 signaling in osteoblasts are likely to augment bone formation and accelerate fracture repair. Therefore, the treatment of negative pressure was hypothesized to be capable of triggering differentiation into osteoblasts.

In order to investigate the effects of the negative pressure on the differentiation of MSCs, ALP activity, type I collagen and VEGF expression were also detected. The results demonstrate that the three factors were enhanced significantly compared with the untreated control group, and promoted and induced differentiation into osteoblasts. This effect of negative pressure may be attributed to mechanical stimulation and cellular hypoxia. Mechanical stimulation plays a basic role in the cell development. Various mechanical stimuli have been revealed to act through numerous signaling pathways that result in changes in gene expression and proliferation of osteoblasts. Previous studies $(20,21)$ have indicated that mechanical stimulation promotes the osteogenic differentiation of human bone marrow stromal cells. Wiesmann et al (22) also reported that the collagen type I and osteonectin expression levels were significantly enhanced by the mechanical stimulation. Compared with other studies, the shorter 30-min intermittent negative pressure treatment time at a frequency of two times per day was hypothesized to increase the expression of type I collagen and VEGF, which are mediated by HIF-1 and promote bone formation.

The present study demonstrated that intermittent negative pressure decreased OPG and OPGL mRNA expression in MSCs. OPG plays a key role in the physiological regulation of osteoclastic bone resorption and acts by binding to OPGL. Negative pressure decreased the ratio of OPG to OPGL in vitro and may therefore correlate with osteogenesis and osteoclastogenesis, which may associate with temporal hypoxia. A pronounced decrease in OPG/OPGL caused by mechanical stimuli results in an increase in bone formation and an inhibition of bone absorption $(23,24)$. The results of the present study revealed a concomitant change of OPG and OPGL mRNA expression in response to a variety of mechanical stimuli. However, these observations are inconsistent with a study by Rubin et al which demonstrated that the change was attributed to decreased mRNA expression of OPGL only.

To investigate the specific mechanism of cell proliferation of negative pressure-treated MSCs, ER stress-associated proteins (UPR pathway) $(25,26)$, including p-Perk, IRE1 and cleaved ATF6 levels, were detected. Three proteins were demonstrated to be activated when treated with negative pressure. Therefore, the UPR pathway may be involved in ER stress-associated apoptosis. Furthermore, the downstream factors, including spliced XBP1, TRAF2 and eIF- $2 \alpha$ proteins were also detected. The activation of Perk may phosphorylate eIF- $2 \alpha$ proteins and the results markedly indicated the emergence of an ER stress (or UPR pathway) following treatment with negative pressure in the MSCs. The observation that treatment of negative pressure triggered ER stress of MSCs 
is of great importance. Therefore we hypothesize that, when treated with negative pressure, the proliferation of the MSCs may be blocked by the ER stress-triggered apoptosis and induce differentiation of MSCs.

In conclusion, treatment with low-intensity and intermittent negative pressure may inhibit proliferation of MSCs and trigger cellular apoptosis, further enhancing osteogenesis activity and inducing differentiation of osteoblasts. The negative pressure may improve bone formation and differentiation by decreasing the ratio of OPGL/OPG mRNA, inducing type I collagen and VEGF expression and ER stress-trigged apoptosis. Therefore, this method may be promising for the treatment of bone regeneration disorders.

\section{References}

1. Kerachian MA, Harvey EJ, Cournoyer D, Chow TY and Séquin C: Avascular necrosis of the femoral head: vascular hypotheses. Endothelium 13: 237-244, 2006.

2. Rodriguez-Merchan $\mathrm{C}$ and Forriol F: Nonunion: general principles and experimental data. Clin Orthop Relat Res 419: 4-12, 2004.

3. Raffl AB: The use of negative pressure under skin flaps after redical mastectomy. Ann Surg 136: 1048, 1952.

4. Deva AK, Buckland GH, Fisher E, et al: Topical negative pressure in wound management. Med J Aust 173: 128-131, 2000.

5. Banwell PE and Musgrave M: Topical negative pressure therapy: mechanisms and indications. Int Wound J 1: 95-106, 2004.

6. Petrie D, Potter M and Banwell P: The management of lower extremity wounds using topical negative pressure. Int J Low Extrem Wounds 2: 198-206, 2003.

7. Kim JW, Lee MS, Lee CH, Kim HY, Chae SU, Kwak HB and Oh J: Effect of interferon- $\gamma$ on the fusion of mononuclear osteoclasts into bone-resorbing osteoclasts. BMB Rep 45: 281-286, 2012.

8. Potter MJ, Banwell P, Baldwin C, Clayton E, Irvine L, Linge C, et al: In vitro optimization of topical negative pressure regimens for antiogenesis into synthetic dermal replacements. Burns 34: 164-174, 2008

9. Brownlow HC, Reed A and Simpson AH: The vascularity of atrophic non-unions. Injury 33: 145-150, 2002.

10. Li RD, Deng ZL, Hu N, Liang X, Liu B, Luo J, et al: Biphasic effects of TGF $\beta 1$ on BMP9-induced osteogenic differention of mesenchymal stem cells. BMB Rep 45: 509-514, 2012.

11. Cao L, Liu G, Gan Y, Fan Q, Yang F, Zhang X, et al: The use of autologous enriched bone marrow MSCs to enhance osteoporotic bone defect repair in long-term estrogen deficient goats. Biomaterials 33: 5076-5084, 2012.
12. Tsai CC, Yew TL, Yang DC, Huang WH and Hung SC: Benefits of hypoxic culture on bone marrow multipotent stromal cell. Am J Blood Res 2: 148-159, 2012.

13. Chen CH, Wang SS, Wei EI, Chu TY and Hsieh PC: Hyaluronan enhances bone marrow cell therapy for myocardial repair after infarction. Mol Ther 21: 670-679, 2013.

14. Wu JY, Wang YH, Wang GJ, Ho ML, Wang CZ, Yeh ML and Chen CH: Low-power GaAIAs laser irradiation promotes the proliferation and osteogenic differentiation of stem cells via IGF1 and BMP2. PLoS One 7: e44027, 2012.

15. Zhu Y, Ouyang Y, Chang Y, Luo C, Xu J, Zhang C and Huang W: Evaluation of the proliferation and differentiation behaviors of mesenchymal stem cells with partially converted borate glass containing different amounts of strontium in vitro. Mol Med Rep 7: 1129-1136, 2013.

16. Rampichová M, Chvojka J, Buzgo M, Prosecká E, Mikeš P, Vysloužilová L, et al: Elastic three-dimensional poly (E-caprolactone) nanofibre scaffold enhances migration, proliferation and osteogenic differentiation of mesenchymal stem cells. Cell Prolif 46: 23-37, 2013

17. Wu C, Rankin EB and Giaccia AJ: Blood and bones: osteoblastic HIF signaling regulates erythropoiesis. Cell Cycle 11: 2221-2222, 2012.

18. Zhou H, Chen ZB, Tian HQ, Xu X, Wang YQ, Xing GQ and Cheng L: Effects of hypoxia-inducible factor $1 \alpha$ on bone conduction impairment in otitis media with effusion. Acta Otolaryngol 132: 938-943, 2012.

19. Towler DA: Vascular biology and bone formation: hints from HIF. J Clin Invest 117: 1477-1480, 2007.

20. Jagodzinski M, Drescher M, Zeichen J, et al: Effects of cyclic longitudinal mechanical strain and dexamethasone on osteogenic differentiation of human bone marrow stromal cells. Eur Cell Mater 7: 35-41, 2004

21. Zhang P, Wu Y, Dai Q, Fang B and Jiang L: p38-MAPK signaling pathway is not involved in osteogenic differentiation during early response of mesenchymal stem cells to continuous mechanical strain. Mol Cell Biochem 378: 19-28, 2013.

22. Wiesmann A, Bühring HJ, Mentrup C and Wiesmann HP: Decreased CD90 expression in human mesenchymal stem cells by applying mechanical stimulation. Head Face Med 2: 8, 2006.

23. Zhang YG, Yang Z, Zhang H, et al: Effect of negative pressure on human bone marrow mesenchymal stem cells in vitro. Connect Tissue Res 51: 14-21, 2010.

24. Kim CH, You L, Yellowley CE and Jacobs CR: Oscillatory fluid flow-induced shear stress decreases osteoclastogenesis through RANKL and OPG signaling. Bone 39: 1043-1047, 2006.

25. Joung KH and Cho SC: Stress responses of neonates related to maternal characteristics. Yonsei Med J 52: 98-103, 2011.

26. Momoi T, Fujita E, Senoo H and Momoi M: Genetic factors and epigenetic factors for autism: endoplasmic reticulum stress and impaired synaptic function. Cell Biol Int 34: 13-19, 2009. 